\title{
Structural Modifications for Torsional Vibration Control of Shafting Systems Based on Torsional Receptances
}

\author{
Zihao Liu, ${ }^{1}$ Wanyou Li, ${ }^{1}$ and Huajiang Ouyang ${ }^{2,3}$ \\ ${ }^{1}$ College of Power and Energy Engineering, Harbin Engineering University, Harbin 150001, China \\ ${ }^{2}$ School of Engineering, University of Liverpool, Liverpool L69 3GH, UK \\ ${ }^{3}$ State Key Laboratory of Structural Analysis of Industrial Equipment, Dalian University of Technology, Dalian 116023, China
}

Correspondence should be addressed to Wanyou Li; hrbeu_ripet_lwy@163.com

Received 28 June 2016; Revised 18 August 2016; Accepted 22 August 2016

Academic Editor: Jussi Sopanen

Copyright (c) 2016 Zihao Liu et al. This is an open access article distributed under the Creative Commons Attribution License, which permits unrestricted use, distribution, and reproduction in any medium, provided the original work is properly cited.

\begin{abstract}
Torsional vibration of shafts is a very important problem in engineering, in particular in ship engines and aeroengines. Due to their high levels of integration and complexity, it is hard to get their accurate structural data or accurate modal data. This lack of data is unhelpful to vibration control in the form of structural modifications. Besides, many parts in shaft systems are not allowed to be modified such as rotary inertia of a pump or an engine, which is designed for achieving certain functions. This paper presents a strategy for torsional vibration control of shaft systems in the form of structural modifications based on receptances, which does not need analytical or modal models of the systems under investigation. It only needs the torsional receptances of the system, which can be obtained by testing simple auxiliary structure attached to relevant locations of the shaft system and using the finite element model (FEM) of the simple structure. An optimization problem is constructed to determine the required structural modifications, based on the actual requirements of modal frequencies and mode shapes. A numerical experiment is set up and the influence of several system parameters is analysed. Several scenarios of constraints in practice are considered. The numerical simulation results demonstrate the effectiveness of this method and its feasibility in solving torsional vibration problems in practice.
\end{abstract}

\section{Introduction}

Dynamic performance of structures plays an important role in engineering; however, there are always some circumstances in which structural dynamic performance does not meet the design requirements or actual situations in practice. Therefore, it is common that some existing structures need to be modified in order to acquire desired dynamic performance [1]. Many researchers have put forward many methods for the eigenstructure assignment problems [2-5]. One major way of doing that is to assign structure suitable natural frequencies and modes through structural modifications as a typical vibration control strategy, which usually requires knowledge of accurate structural parameters (e.g., mass, stiffness, and damping matrices $[6,7]$ ) or modal data [8-10]. However, in most engineering problems, it is very difficult to gain such knowledge. Usually modal tests must be conducted [11] and model updating must be carried out [12], which is expensive and tedious for complicated structures. Moreover, the application of modal data in practice has a number of difficulties, which was discussed in [13]. On the other hand, some structural modification methods directly based on the measured system receptances or Frequency Response Functions (FRFs) [14-17] overcome those difficulties and provide effective solutions to this kind of problems, which belong to inverse structural dynamics. Structural modifications based on the measured receptance or FRFs were studied in forward analysis for prediction of receptances of the modified structure [18] and in inverse analysis for assigning natural frequencies and vibration nodes [19] and eigenstructures $[13,20]$.

For rotating machines, one of the most important problems is torsional vibration of shafts. The adverse impact caused by torsional vibration includes vibration of the whole machine, damage in the transmission system, excessive wear of bearings and gears, and even shaft fracture [21]. For shaft structures, numerical models are usually needed to evaluate torsional vibration characteristics in the engineering design 
stage, but some structural parameters (such as the rotary inertia of the motor and the actual torsional stiffness of gears) cannot be accurately obtained easily. Therefore, there are inevitably considerable discrepancies between the designs and the actual structures based on such imperfect theoretical models. So suppression of torsional vibration is a big challenge. If there is a method that does not require an accurate theoretical system model in solving the torsional vibration problems and can also achieve structural modifications to the system based on measured data, this method will bring many advantages in practice.

However, it should be noted that, perhaps partly owing to difficulties in accurately measuring torsional FRFs, inverse structural dynamics problems of rotating machineries based on measured data (especially for rotational receptance) nearly have never been studied before [22].

Although many researchers have put forward a number of methods for measuring transfer functions for rotational degrees of freedom (DoFs); for example, Mottershead et al. [23] proposed one indirect method based on T-block for obtaining rotational receptances, the progress in measuring torsional transfer functions in shaft systems is still very limited [23-27], and nearly none of these papers are about torsional vibration measurement of shaft structures. Recently, Lv et al. [22] put forward an indirect method to measure the torsional receptance. The method was implemented by using a T-shaped simple auxiliary structure attached to one end of the shaft system, and the torsional system receptances could be obtained accurately through combining the auxiliary structure's finite element model (FEM) and test data of the whole structure.

This paper presents a theoretical strategy of structural modifications for suppression of torsional vibration of shaft systems, using "measured" torsional receptances and a structural optimization method. One main advantage of this method proposed in this paper is that it does not need any knowledge of mass and stiffness parameters or even analytical or modal models of the system under investigation; instead "measured" torsional receptance data are used, which can be obtained from measured translational vibration data obtained through an additional structure. In this paper, structural modifications for suppressing torsional vibration of a simplified model of a "real" rotating machine are studied. Several scenarios of practical constraints are considered. Theoretical results show the effectiveness of this method.

\section{Receptance-Based Method}

A shaft system under a harmonic excitation, treated as a general linear discrete conservative dynamic system without damping, can be described by

$$
\mathbf{J} \ddot{\mathbf{x}}+\mathbf{K x}=\mathbf{f} \mathrm{e}^{i \omega t}
$$

where $\mathbf{J}$ is the mass (moment of inertia) matrix, $\ddot{\mathbf{x}}$ is the acceleration vector, $\mathbf{K}$ is the stiffness matrix, $\mathbf{x}$ is the displacement vector, $\mathbf{f}$ is the force amplitude vector, $\mathrm{e}$ is the Euler number, $i=\sqrt{-1}, \omega$ is the angular velocity, and $t$ is the time variable.
Denote the change in mass matrix and change in stiffness matrix due to structural modifications as $\delta \mathbf{J}$ and $\delta \mathbf{K}$, respectively. Then (1) becomes

$$
(\mathbf{J}+\delta \mathbf{J}) \ddot{\mathbf{x}}+(\mathbf{K}+\delta \mathbf{K}) \mathbf{x}=\mathbf{f} \mathrm{e}^{i \omega t} .
$$

It can be assumed that the response is harmonic in the form of $\mathbf{x}=\mathbf{u} \mathrm{e}^{i \omega t}$; then $\mathbf{u}$ is the eigenvector. Substituting it into (2) yields

$$
\left(-\omega^{2} \mathbf{J}+\mathbf{K}\right) \mathbf{u}=\left(\omega^{2} \delta \mathbf{J}-\delta \mathbf{K}\right) \mathbf{u}+\mathbf{f}
$$

The original system FRF matrix is defined as $\mathbf{H}(\omega)=\left(-\omega^{2} \mathbf{J}+\right.$ $\mathbf{K})^{-1}$. Equation (3) can then be rewritten as

$$
\mathbf{H}^{-1} \mathbf{u}=\left(\omega^{2} \delta \mathbf{J}-\delta \mathbf{K}\right) \mathbf{u}+\mathbf{f} .
$$

For the eigenvalue problem, it is assumed that the desired natural frequency and mode are, respectively, $\omega_{h}$ and $\mathbf{u}_{h}$; then the following equation is derived:

$$
\mathbf{H}^{-1}\left(\omega_{h}\right) \mathbf{u}_{h}=\left(\omega_{h}^{2} \delta \mathbf{J}-\delta \mathbf{K}\right) \mathbf{u}_{h} .
$$

In order to obtain the FRFs of the unknown shaft system, a simple auxiliary structure needs to be attached to the shaft at one end, as shown in Figure 1.

Next, the T-shaped simple auxiliary structure is divided into two parts to be considered. One part is the very short OD structure whose torsional vibration about $z$-axis (not including beam AOB) is only considered; the other part is beam AOB whose bending vibration is only considered. A good FE model of auxiliary structure must be established, which is fairly easy, given its simple geometric shape. The whole derivation details are given in Lv et al.s paper [22].

Firstly, the force analysis for torsional vibration is done on the OD structure, and the shaft system receptances at point $D$ are given in [22] by

$$
H_{\theta_{D} \theta_{D}}(\omega)=\frac{\widehat{H}_{\theta_{O} \theta_{D}}(\omega) \widehat{H}_{\theta_{D} \theta_{O}}(\omega)}{\widehat{H}_{\theta_{O} \theta_{O}}(\omega)-H_{\theta_{O} \theta_{O}}(\omega)}-\widehat{H}_{\theta_{D} \theta_{D}}(\omega),
$$

where subscript $\theta$ is to show that the FRF $\mathbf{H}$ is for the freedom of rotation and the subscripts $D$ and $O$ mean the points on the T-shaped simple auxiliary structure, as shown in Figure 1.

Next step is to consider only the bending vibration of the AOB beam. By taking $n$ times average of the test results of two different loading conditions (from points $A$ and $B$ ), an estimation formula of $\mathbf{H}_{\mathrm{OO}}(\omega)$ could be described by

$$
\mathbf{H}_{\mathrm{OO}}(\omega)=\mathbf{B}(\omega) \mathbf{A}^{-1}(\omega)
$$

where

$$
\begin{aligned}
& \mathbf{A}(\omega)=\left[\begin{array}{ll}
\mathbf{R}(\omega) & \mathbf{S}(\omega)
\end{array}\right]\left[\begin{array}{ll}
\mathbf{G}_{x_{2} x_{2}} & \mathbf{G}_{x_{2} f_{2}} \\
\mathbf{G}_{f_{2} x_{2}} & \mathbf{G}_{f_{2} f_{2}}
\end{array}\right]\left[\begin{array}{l}
\mathbf{R}^{* T}(\omega) \\
\mathbf{S}^{* T}(\omega)
\end{array}\right], \\
& \mathbf{B}(\omega)=\left[\begin{array}{ll}
\mathbf{T}(\omega) & \mathbf{U}(\omega)
\end{array}\right]\left[\begin{array}{ll}
\mathbf{G}_{x_{2} x_{2}} & \mathbf{G}_{x_{2} f_{2}} \\
\mathbf{G}_{f_{2} x_{2}} & \mathbf{G}_{f_{2} f_{2}}
\end{array}\right]\left[\begin{array}{l}
\mathbf{R}^{* T}(\omega) \\
\mathbf{S}^{* T}(\omega)
\end{array}\right]
\end{aligned}
$$




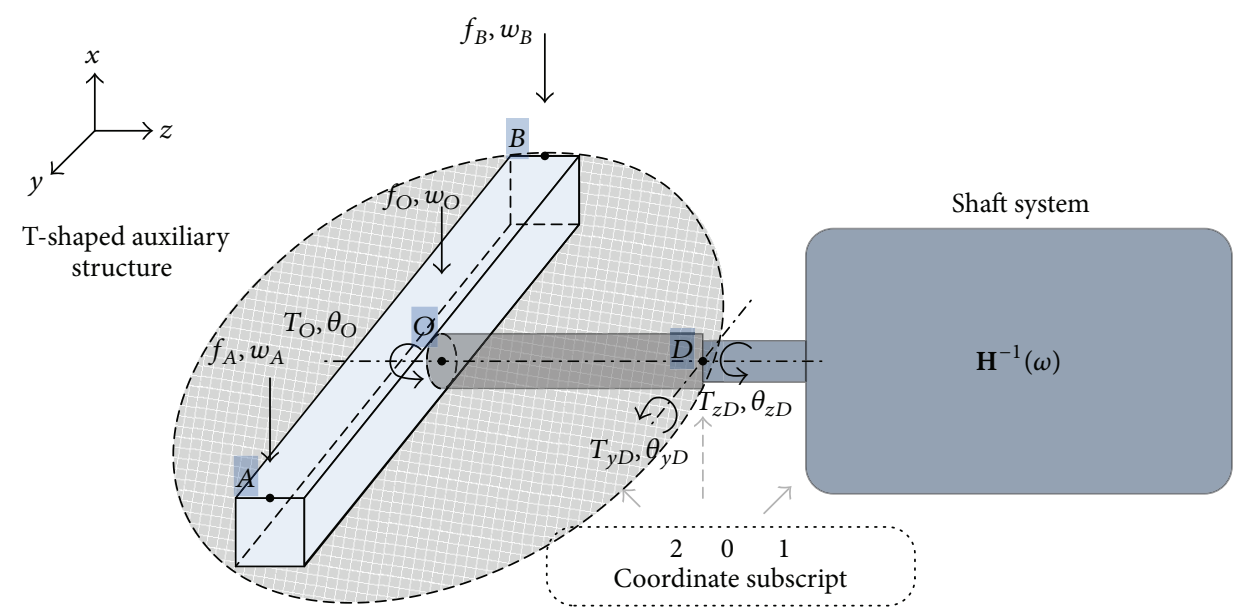

FIGURE 1: The schematic of the auxiliary structure and the shaft structure under study (not in scale).

and matrix $\mathbf{G}$ is autospectrum or cross spectrum of the excitation and response. Taking the submatrix $\mathbf{G}_{x_{2} f_{2}}(\omega)$ as an example, it could be obtained by the following formula:

$$
\mathbf{G}_{x_{2} f_{2}}(\omega)=\sum_{1}^{2} \frac{1}{n} \sum_{i=1}^{n}\left[\begin{array}{ll}
x_{A}^{i} f_{A}^{i} & x_{A}^{i} f_{B}^{i} \\
x_{B}^{i} f_{A}^{i} & x_{B}^{i} f_{B}^{i}
\end{array}\right]
$$

where $x$ stands for the measured response signal data, $f$ stands for the measured excitation signal data, the subscript number 2 means the T-shaped simple auxiliary structure, shown in Figure 1, and $n$ stands for the number of the data. Further details about the derivation of (7) can be found in [22].

By substituting $\mathbf{H}_{\mathrm{OO}}$ into (6), the needed torsional receptances $H_{\theta_{D} \theta_{D}}$ are found. Therefore, (5) can be rewritten as

$$
\mathbf{u}_{h}=\mathbf{H}\left(\omega_{h}\right)\left(\omega_{h}^{2} \delta \mathbf{M}-\delta \mathbf{K}\right) \mathbf{u}_{h} .
$$

For the sake of convenience in presenting the method and without losing generality, it is assumed that the structural modifications are to be made to the last $m$ DoFs. Then $\delta \mathbf{M}$ and $\delta \mathbf{K}$ have only nonzero elements in those rows and columns corresponding to the modified DoFs. As a result,

$$
\mathbf{v}=\left(\omega_{h}^{2} \delta \mathbf{M}-\delta \mathbf{K}\right)\left\{\begin{array}{c}
0 \\
0 \\
\vdots \\
0 \\
0 \\
u_{1} \\
\vdots \\
u_{m}
\end{array}\right\},
$$

where $u$ denotes nonzero terms, which correspond to the modified DoFs and involve the products of the modifications and modal displacements at only those modified DoFs.
Consequently, the $n$th row of (10) can be written as

$$
\left(u_{h}\right)_{n}=\sum_{j=1}^{m}(H)_{n j}(v)_{j},
$$

where $m$ denotes the modified (last few) DoFs.

Equation (12) is worth a close examination. If certain $n$ modal displacements of a mode are to be assigned certain values, (1) reveals that only $\mathbf{H}$ elements in those $n$ rows and $j$ columns are required, which means only $n \times j$ number of $\mathbf{H}$ elements, which can be a very small number even for a very complicated structure. $j$ should be those locations where structural modifications are allowed and easy to do, while $n$ should be either some interesting locations of the structure, where the modal displacements need to take certain specified values, or any convenient DoFs if there are not particular interesting locations. For different modes, assigned modal displacements could even be at different locations. It should be pointed out that, for certain assigned frequencies and associated modes, there is no guarantee that there is a solution or a unique solution.

The eigenstructure assignment problem can finally be cast as

$$
\min _{\mathbf{x}}\left\{\sum_{h=1}^{n} \alpha_{h}\left\|\mathbf{H}\left(\omega_{h}\right)\left(\omega_{h}^{2} \delta \mathbf{M}-\delta \mathbf{K}\right) \mathbf{u}_{h}-\mathbf{u}_{h}\right\|_{2}^{2}\right\}
$$

where $\alpha_{h}$ is the weighting coefficient (a positive scalar).

Equation (13) can be solved by optimization algorithms, which have already been applied in various fields [28]. It should be noted that the focus of this paper is to establish a novel strategy for reducing torsional vibration of shaft structures through structural modifications and show its effectiveness. Hence, the algorithms for solving this optimization problem will not be studied in this paper.

The proposed receptance-based strategy does not require information of the mass or stiffness matrices of the original system, and it only needs a few receptances that can be measured relatively easily, for example, by using the method presented in [22]. This method is particularly suitable in 


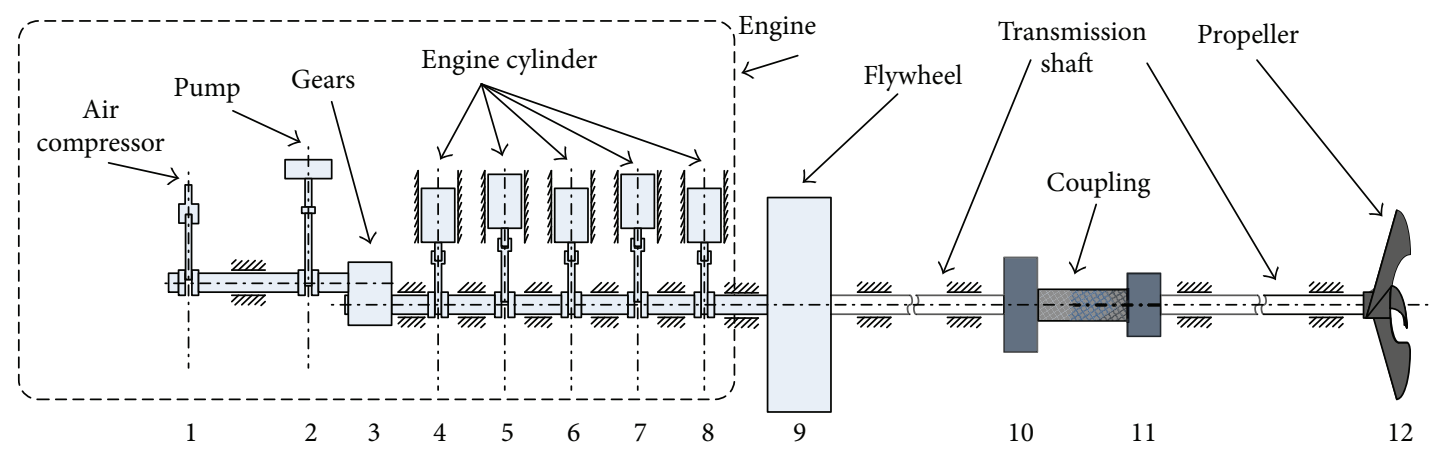

FIgURE 2: Schematic diagram of the simulated 12-DoF system.

TABLE 1: System parameters.

\begin{tabular}{lccc}
\hline Parameter & Value $\left[\mathrm{kg} \cdot \mathrm{m}^{2}\right]$ & Parameter & Value $\left[10^{5} \mathrm{~N} \cdot \mathrm{m}\right]$ \\
\hline$J_{1}{ }^{*}$ & 5 & $k_{12}{ }^{*}$ & 10 \\
$J_{2}{ }^{*}$ & 2 & $k_{23}{ }^{*}$ & 200 \\
$J_{3}{ }^{*}$ & 1 & $k_{34}$ & 150 \\
$J_{4}$ & 3 & $k_{45}$ & 100 \\
$J_{5}$ & 3 & $k_{56}$ & 100 \\
$J_{6}$ & 3 & $k_{67}$ & 100 \\
$J_{7}$ & 3 & $k_{78}$ & 100 \\
$J_{8}$ & 3 & $k_{89}$ & 200 \\
$J_{9}$ & 40 & $k_{9,10}$ & 7 \\
$J_{10}$ & 5.5 & $k_{1,11}$ & 4 \\
$J_{11}$ & 3.5 & $k_{11,12}$ & 50 \\
$J_{12}$ & 8 & & \\
\hline
\end{tabular}

Note: Because there are gears in the system, superscript $*$ means that this parameter is an equivalent value after considering the influence of gear ratios, which are calculated by real values times the gear ratios.

dealing with those shaft systems that suffer from torsional vibration problems in which mass (moment of inertia) and stiffness matrices are difficult to measure.

\section{Numerical Experiment}

The shaft modification method proposed in this paper is based on "measured" torsional receptances. During the numerical simulation stage, a shaft system is chosen as an example of the numerical experiments to verify the feasibility of the method proposed. The required data, torsional system receptances of the shaft, are obtained directly from the system parameters in the simulation.

3.1. Model Setup. The model reflects a ship propulsion shafting, including air compressors, pumps, cylinders, flywheels, couplings, propeller, and other components in series, and the whole structure is shown in Figure 2.

According to the structure and properties of materials, the model is simplified to get each structural inertia and torsional stiffness, as shown in Table 1 . The natural frequencies of the original system are given in Table 2, and the first and the third modes are shown in Figure 3.
TABLE 2: Original system modes.

\begin{tabular}{lccccc}
\hline Mode number & 1 & 2 & 3 & 4 & 5 \\
\hline$f[\mathrm{~Hz}]$ & 24.96 & 57.52 & 74.65 & 108.25 & 232.69 \\
\hline
\end{tabular}

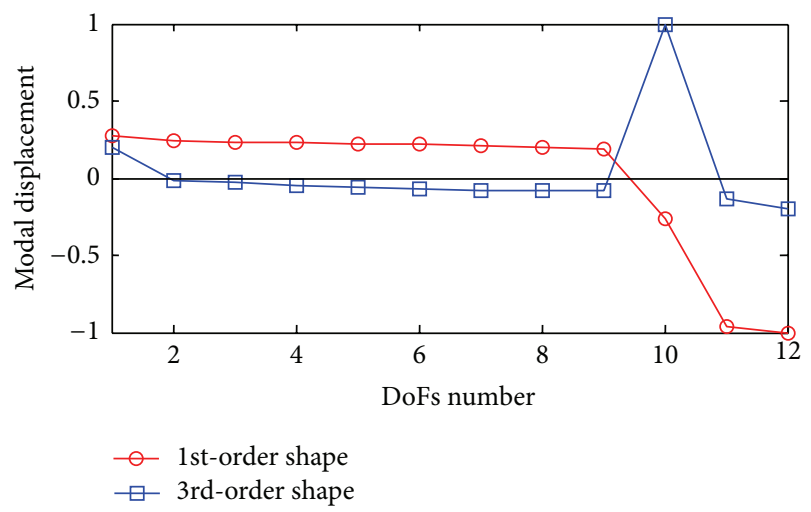

FIgURE 3: Original system's 1st-order and 3rd-order modes shapes.

For the numerical application of the proposed method, the system FRFs are acquired by solving matrix $\left[\omega_{h}{ }^{2} \mathbf{M}-\right.$ $\mathbf{K}]^{-1}$ at desired frequencies of $\omega_{1}$ and $\omega_{3}$ (they are two safe frequencies away from resonance, 30 and $90 \mathrm{~Hz}$, resp., and the reason for choosing the values will be explained in the next subsection). The 2 nd natural frequency $\omega_{2}$ does not need to be shifted, which does not have high risks of resonance when the shaft is working in the rated rotation speed.

3.2. Modification Goals. It is assumed that the rated speed of the shaft system is $1500 \mathrm{r} / \mathrm{min}$, so that $25 \mathrm{~Hz}$ is the main shafting vibration excitation frequency. Moreover, excitation from subharmonics and superharmonics of the rated rotation speed $(0.5,1$, and 2 times of $25 \mathrm{~Hz})$, shown by red dotted lines in Figure 4, is also possible. In addition, for the 3-blade propeller, another main excitation frequency is three times of $25 \mathrm{~Hz}$. It is important to ensure that the natural frequencies of the structure do not coincide with the excitation frequencies [29]. So there are at least two must-avoid frequencies, $25 \mathrm{~Hz}$ and $75 \mathrm{~Hz}$, shown with thicker red dotted lines in Figure 4.

During its run-up, the shaft system needs to go through a speed-increasing process. If some natural frequencies are 
TABLE 3: Modification bounds.

\begin{tabular}{lccc}
\hline & Parameter & Lower bound & Upper bound \\
\hline Moment of inertia $\left[\mathrm{kg} \cdot \mathrm{m}^{2}\right]$ & $J_{10}$ & -0.5 & 0 \\
& $J_{11}$ & -1 & 0 \\
\hline Stiffness $[\mathrm{kN} \cdot \mathrm{m}]$ & $k_{10,11}$ & 0 & 500 \\
\hline & Desired mode number, $h$ & $u_{h, 10}$ & $u_{h, 11}$ \\
\hline Desired modal displacements & 1 & -0.4765 & -0.9432 \\
& 3 & 1 & -0.1513 \\
\hline
\end{tabular}

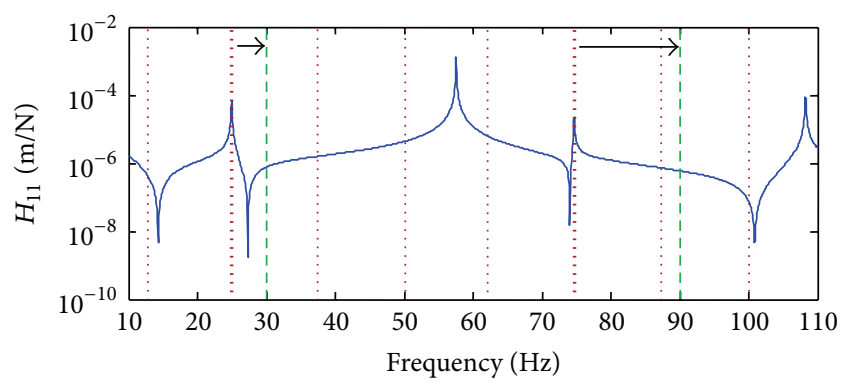

FIGURE 4: FRF with desired frequencies.

lower than the rotation frequency, then these frequencies will be excited, which may lead to the resonance of the shaft and bring about some serious damage.

Therefore, considering both points above, the first natural frequency of the shaft is preferably greater than $25 \mathrm{~Hz}$. Therefore, the modification goal is to shift the 1st and 3rd natural frequencies to $30 \mathrm{~Hz}$ and $90 \mathrm{~Hz}$, respectively, as shown by the green dashed lines in Figure 4, which aims to move the system away from these potentially damaging frequencies to two "safe" frequencies. In addition, the 2 nd natural frequency $(57.52 \mathrm{~Hz})$ is not located around any harmonic frequencies of the basic frequency, $25 \mathrm{~Hz}$, so it is not necessary to modify it. In this example, because there are no special requirements on mode shapes, for simplicity, the mode shapes, as shown in Figure 3, remain unchanged.

Another main reason for using the original modes as the desired modes is that, after moving the natural frequencies to a safe region, vibration response becomes much reduced and mode shapes are no longer a concern. In the event that certain modal displacement should take some desirable values, the method presented in this paper is equally applicable. This will be demonstrated by an example.

In addition, the method proposed especially suits for assigning only a few modal displacements for one mode; on the other hand, a whole mode also could be assigned if receptances at all these DoFs are available, which means that more test data are required. The method proposed in this paper theoretically is fully capable of assigning any frequencies and modes for vibration reduction, which will be proven by another example.

\section{Results}

In comparison with other complicated structures (such as engines, air compressors, and pumps), couplings are easy to

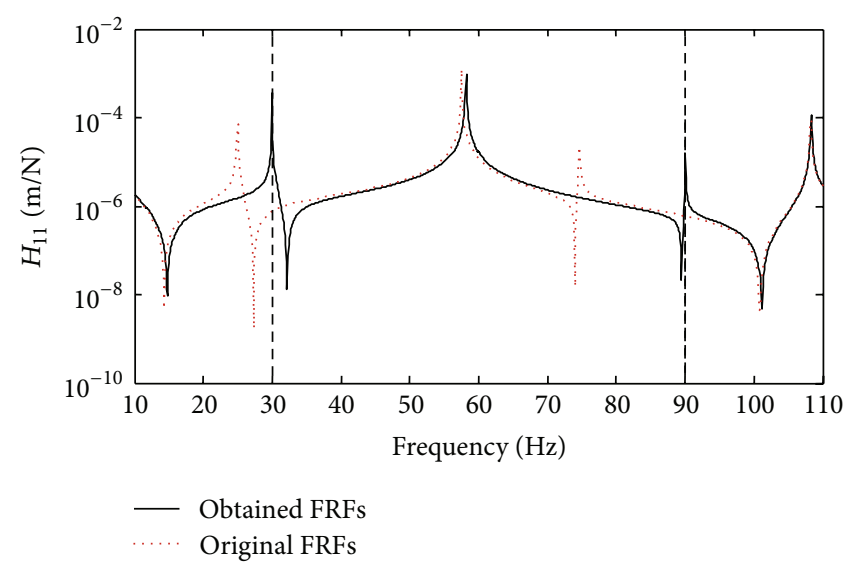

FIGURE 5: Frequency response comparison.

be replaced in shaft systems and there are many types. In other words, for complicated equipment (e.g., an engine), once the type has been decided upon in the design stage of a shaft system, it would be very difficult or unrealistic to modify any inertia or stiffness of it. Thus, in this paper, the chosen specific strategy is to modify the coupling, which contains two moments of inertia and torsional stiffness between them [30].

In this paper, the optimization problem is solved by a genetic algorithm. Other effective optimization algorithms can also be used.

4.1. Assignment of Frequencies and Part of Modes. As the method proposed in Section 2, aiming at only considering 10th and 11th DoFs, the modified parameters are $J_{10}, J_{11}$, and $k_{10,11}$. In other words, the subscripts $i$ and $j$ in (12) are equal to either 10 and 11, and $\mathbf{H}$ elements only in those $i$ rows and $j$ columns are required. For convenience of mathematical treatment and computer coding, these two DoFs are moved to the last two elements of $\mathbf{v}$ (see (11)). But for the description of the process of using this method, there is no need to do so.

For this optimization problem, the modification bounds and desired values of modal displacements are listed in Table 3.

After solving the optimization problem, a solution for structural modifications in the form of a group of two moments of inertia and stiffness is obtained, as shown in Table 4, and the system FRFs, $H_{11}$, are shown in Figure 5, respectively. The difference between the desired natural frequencies and obtained frequencies is shown as $\left|f_{h}-f_{i}\right|$ in 
TABLE 4: Obtained frequencies and required structural modifications.

\begin{tabular}{|c|c|c|c|c|c|}
\hline & & & Parameter & Initial value & Obtained value \\
\hline \multirow{2}{*}{ Moment of inertia $\left[\mathrm{kg} \cdot \mathrm{m}^{2}\right]$} & & & $J_{10}$ & 5.5 & 5.15 \\
\hline & & & $J_{11}$ & 3.5 & 2.65 \\
\hline \multirow[t]{2}{*}{ Stiffness $[\mathrm{kN} \cdot \mathrm{m}]$} & & & $k_{10,11}$ & 400 & 800.01 \\
\hline & & Desired mode number, $h$ & Goal & Proposed method & Error \\
\hline \multirow{2}{*}{$\left|f_{h}-f_{i}\right|[\mathrm{Hz}]$} & & 1 & 30 & 30.01 & 0.01 \\
\hline & & 3 & 90 & 90.02 & 0.02 \\
\hline \multirow{2}{*}{$\mathbf{u}_{h}-\mathbf{u}_{i}$} & \multirow{2}{*}{1} & $u_{1,10}$ & -0.4765 & -0.4766 & 0.0001 \\
\hline & & $u_{1,11}$ & -0.9432 & -0.9431 & -0.0001 \\
\hline \multirow{2}{*}{$\mathbf{u}_{h}-\mathbf{u}_{i}$} & \multirow{2}{*}{3} & $u_{3,10}$ & 1 & 1 & 0 \\
\hline & & $u_{3,11}$ & -0.1513 & -0.1512 & -0.0001 \\
\hline
\end{tabular}

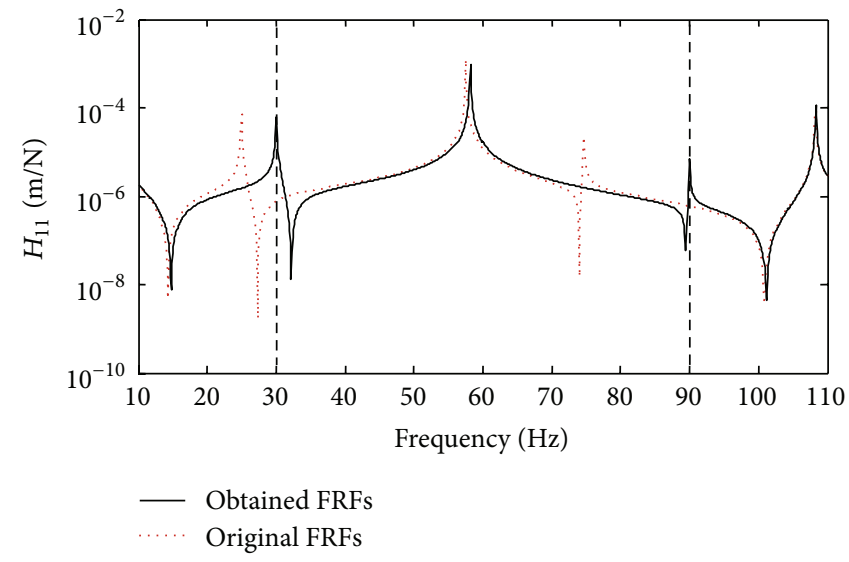

(a)

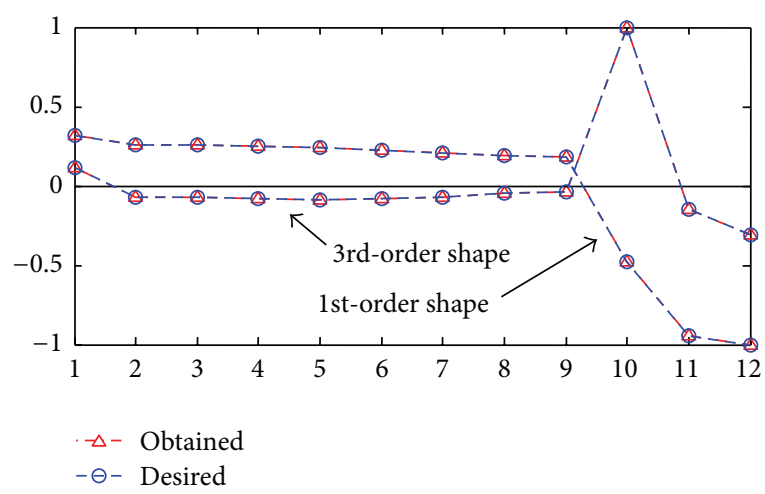

(b)

FIGURE 6: (a) Frequency response comparison. (b) Mode shape comparison.

Table 4, and the differences between desired $\mathbf{u}_{h}$ and obtained $\mathbf{u}_{i}$ are also listed in the last two lines.

It can clearly be seen from the results that the performance of the method proposed is excellent for achieving the desired modal behaviour for this shaft system: the 1st and 3rd natural frequencies of the original shaft system are now shifted to $30 \mathrm{~Hz}$ and $90 \mathrm{~Hz}$ and four modal displacements are assigned. Based on the theory described by (11) and (12), an impressive advantage is that only 4 pieces of "experimental" data of receptances are used in this example, which means several benefits for real engineering applications.

4.2. Assignment of Whole Modes. If whole $\mathbf{u}_{h}$ needs to be assigned, the receptance-based structural modification method is also applicable. In this case, a whole column of matrix $\mathbf{H}$ in (12) is required, that is, in the same size as vector $\mathbf{u}_{h}$. This means that more receptance data would be needed, which would bring a large amount of measurement work in practice. However, in this paper, this is not an issue in this section, as the purpose is to demonstrate its power in assigning whole modes.

The whole modal shapes are calculated by using obtained system parameters in Section 4.1, taken as the desired modes in this section to ensure that the optimization problem has
TABle 5: Modification bounds.

\begin{tabular}{lccc}
\hline & Parameter & Lower bound & Upper bound \\
\hline Moment of & $J_{10}$ & -1 & 0 \\
inertia $\left[\mathrm{kg} \cdot \mathrm{m}^{2}\right]$ & $J_{11}$ & -1 & 0 \\
\hline Stiffness $[\mathrm{kN} \cdot \mathrm{m}]$ & $k_{10,11}$ & 0 & 500 \\
\hline
\end{tabular}

at least one group of solution. The modification bounds and desired modes are listed in Table 5.

The solution for structural modifications obtained from the optimization method, as shown in Table 6, and receptance $H_{11}$ and the mode shapes of the modified system are shown in Figures 6(a) and 6(b), respectively. The difference between the desired natural frequencies and obtained frequencies is shown as $\left|f_{h}-f_{i}\right|$ in Table 6 . It can clearly be seen from the results that the performance of the method proposed is excellent for achieving the desired modal behaviour for this shaft system: the 1st and 3rd natural frequencies of the original shaft system are now shifted to $30 \mathrm{~Hz}$ and $90 \mathrm{~Hz}$; and the obtained mode shapes are nearly the same as the desired ones. The results prove that the proposed method is also capable of assigning whole mode shapes through modifications at only a few DoFs. 
TABLE 6: Obtained frequencies and required structural modifications.

\begin{tabular}{|c|c|c|c|c|}
\hline & & Parameter & Initial value & Obtained value \\
\hline \multirow{2}{*}{ Moment of inertia $\left[\mathrm{kg} \cdot \mathrm{m}^{2}\right]$} & & $J_{10}$ & 5.5 & 5.16 \\
\hline & & $J_{11}$ & 3.5 & 2.60 \\
\hline \multirow[t]{2}{*}{ Stiffness $[\mathrm{kN} \cdot \mathrm{m}]$} & & $k_{10,11}$ & 400 & 800.00 \\
\hline & Desired mode number, $h$ & Goal & Proposed method & Error \\
\hline \multirow{2}{*}{$\left|f_{h}-f_{i}\right|[\mathrm{Hz}]$} & 1 & 30 & 30.05 & 0.05 \\
\hline & 3 & 90 & 89.95 & 0.05 \\
\hline
\end{tabular}

It should be pointed out that, for the proposed method, there is a possibility that an optimal solution under certain constraints (such as the bounds of certain system parameters) may not exist. On the other hand, it is also likely to get multiple optimal solutions in some cases $[13,20]$. The latter means that when choosing different desired shape values in the needed DoFs in mode vector $\mathbf{u}_{h}$, the proposed strategy may provide a variety of good modification schemes, which may bring about significant advantages in practical applications.

\section{Conclusions}

For rotating machines, torsional vibration of shafts is one of the most important problems. One major barrier in vibration reduction is the difficulty in accurately obtaining structural parameters of components (moment of inertia and torsional stiffness) or system FRFs data for a shaft system in practice. A powerful method for vibration reduction is structural modifications based on measured receptances. One recently proposed method provides an indirect way of measuring torsional receptances of shaft systems and provides the required data for receptance-based structural modifications.

This paper presents a strategy of structural modifications for suppressing torsional vibration of shaft systems through assigning desired frequencies and modes, based on a recently proposed method of measuring torsional receptances and structural optimization. It needs only a few receptances that can be measured relatively easily whose number is equal to the number of assigned modal displacements. In this paper, the assignment is formulated as a structural optimization problem and a numerical experiment is conducted. Since some parameters of some complicated components of shaft systems (e.g., the engine, compressor, and pumps) commonly are not allowed to be modified in practice, the chosen strategy is to modify the coupling (which contains two moments of inertia and torsional stiffness in between), which is easy to be replaced in shaft systems and there are many types. Under certain reasonable bounds, the numerical simulation leads to two sets of good results: the 1st and 3rd natural frequencies $(25 \mathrm{~Hz}$ and $75 \mathrm{~Hz})$ of the original system are both accurately shifted to two "safe" frequencies $(30 \mathrm{~Hz}$ and $90 \mathrm{~Hz}$ ), respectively; two modal displacements of the corresponding modes and the wholes of the corresponding modes are also accurately assigned. It is thus proven that the proposed method is excellent for achieving the desired modal behaviour and provides a valid solution to suppress torsional vibration for shaft systems in engineering.

\section{Competing Interests}

The authors declare that there are no competing interests regarding the publication of this paper.

\section{Acknowledgments}

This work is partly funded by the National Natural Science Foundation of China (no. 51375104) and "the Fundamental Research Funds for the Central Universities" (DUT16RC(3)027) and is supported by China Scholarship Council (CSC). The main part of the theoretical work was carried out at the School of Engineering, University of Liverpool, during the year-long visit by the first author under the supervision of the third author.

\section{References}

[1] M. Rastgaar, M. Ahmadian, and S. Southward, "A review on eigenstructure assignment methods and orthogonal eigenstructure control of structural vibrations," Shock and Vibration, vol. 16, no. 6, pp. 555-564, 2009.

[2] B. N. Datta, "Finite-element model updating, eigenstructure assignment and eigenvalue embedding techniques for vibrating systems," Mechanical Systems and Signal Processing, vol. 16, no. 1, pp. 83-96, 2002.

[3] F. Blanchini, "Eigenvalue assignment via state observer for descriptor systems," Kybernetika, vol. 27, no. 4, pp. 384-392, 1991.

[4] B. N. Datta and D. Sarkissian, "Theory and computations of some inverse eigenvalue problems for the quadratic pencil," Contemporary Mathematics, vol. 280, pp. 221-240, 2001.

[5] M. J. Triller and D. C. Kammer, "Improved eigenstructure assignment controller design using a substructure-based coordinate system," Journal of Guidance, Control, and Dynamics, vol. 20, no. 5, pp. 941-948, 1997.

[6] A. Kyprianou, J. E. Mottershead, and H. Ouyang, "Assignment of natural frequencies by an added mass and one or more springs," Mechanical Systems and Signal Processing, vol. 18, no. 2, pp. 263-289, 2004.

[7] D. Richiedei, A. Trevisani, and G. Zanardo, "A constrained convex approach to modal design optimization of vibrating systems," Journal of Mechanical Design, vol. 133, no. 6, Article ID 061011, 2011.

[8] I. Bucher and S. Braun, "The structural modification inverse problem: an exact solution," Mechanical Systems and Signal Processing, vol. 7, no. 3, pp. 217-238, 1993. 
[9] S. G. Braun and Y. M. Ram, "Modal modification of vibrating systems: some problems and their solutions," Mechanical Systems and Signal Processing, vol. 15, no. 1, pp. 101-119, 2001.

[10] D. D. Sivan and Y. M. Ram, "Optimal construction of a massspring system with prescribed modal and spectral data," Journal of Sound and Vibration, vol. 201, no. 3, pp. 323-334, 1997.

[11] L. M. Crocker and J. Algina, Introduction to Classical and Modern Test Theory, JSTOR Press, 1986.

[12] M. Friswell and J. E. Mottershead, Finite Element Model Updating in Structural Dynamics, Springer Science \& Business Media Press, 1995.

[13] H. Ouyang, D. Richiedei, A. Trevisani, and G. Zanardo, "Eigenstructure assignment in undamped vibrating systems: a convexconstrained modification method based on receptances," Mechanical Systems and Signal Processing, vol. 27, no. 1, pp. 397409, 2012.

[14] J. E. Mottershead, "Structural modification for the assignment of zeros using measured receptances," Journal of Applied Mechanics, vol. 68, no. 5, pp. 791-798, 2001.

[15] A. Kyprianou, J. E. Mottershead, and H. Ouyang, "Structural modification. Part 2: assignment of natural frequencies and antiresonances by an added beam," Journal of Sound and Vibration, vol. 284, no. 1-2, pp. 267-281, 2005.

[16] H. Ouyang, "Prediction and assignment of latent roots of damped asymmetric systems by structural modifications," Mechanical Systems and Signal Processing, vol. 23, no. 6, pp. 1920-1930, 2009.

[17] Y.-H. Park and Y.-S. Park, "Structural modification based on measured frequency response functions: an exact eigenproperties reallocation," Journal of Sound and Vibration, vol. 237, no. 3, pp. 411-426, 2000.

[18] H. N. Özgüven, "Structural modifications using frequency response functions," Mechanical Systems and Signal Processing, vol. 4, no. 1, pp. 53-63, 1990.

[19] J. Mottershead, C. Mares, and M. Friswell, "An inverse method for the assignment of vibration nodes," Mechanical Systems and Signal Processing, vol. 15, no. 1, pp. 87-100, 2001.

[20] Z. Liu, W. Li, H. Ouyang, and D. Wang, "Eigenstructure assignment in vibrating systems based on receptances," Archive of Applied Mechanics, vol. 85, no. 6, pp. 713-724, 2015.

[21] D. N. Walker, Torsional Vibration of Turbomachinery, McGrawHill Professional Press, 2004.

[22] B. Lv, H. Ouyang, W. Li, Z. Shuai, and G. Wang, "An indirect torsional vibration receptance measurement method for shaft structures," Journal of Sound and Vibration, vol. 372, pp. 11-30, 2016.

[23] J. E. Mottershead, A. Kyprianou, and H. Ouyang, "Structural modification. Part 1: rotational receptances," Journal of Sound and Vibration, vol. 284, no. 1-2, pp. 249-265, 2005.

[24] J. E. Mottershead, M. G. Tehrani, D. Stancioiu, S. James, and H. Shahverdi, "Structural modification of a helicopter tailcone," Journal of Sound and Vibration, vol. 298, no. 1-2, pp. 366-384, 2006.

[25] J. E. Mottershead and Y. M. Ram, "Inverse eigenvalue problems in vibration absorption: passive modification and active control," Mechanical Systems and Signal Processing, vol. 20, no. 1, pp. 5-44, 2006.

[26] H. Kim, C. I. Park, S. H. Lee, and Y. Y. Kim, "Non-contact modal testing by the electromagnetic acoustic principle: applications to bending and torsional vibrations of metallic pipes," Journal of Sound and Vibration, vol. 332, no. 4, pp. 740-751, 2013.
[27] M. L. M. Duarte and D. J. Ewins, "Rotational degrees of freedom for structural coupling analysis via finite-difference technique with residual compensation," Mechanical Systems and Signal Processing, vol. 14, no. 2, pp. 205-227, 2000.

[28] X. Xu and Y. Luo, "Form-finding of nonregular tensegrities using a genetic algorithm," Mechanics Research Communications, vol. 37, no. 1, pp. 85-91, 2010.

[29] M. S. Djoudi, H. Bahai, and I. I. Esat, "An inverse eigenvalue formulation for optimizing the dynamic behaviour of pinjointed structures," Journal of Sound and Vibration, vol. 253, no. 5, pp. 1039-1050, 2002.

[30] W. Li, Z. Chai, M. Wang, X. Hu, and Y. Guo, "Online identification and verification of the elastic coupling torsional stiffness," Shock and Vibration, vol. 2016, Article ID 2016432, 7 pages, 2016. 


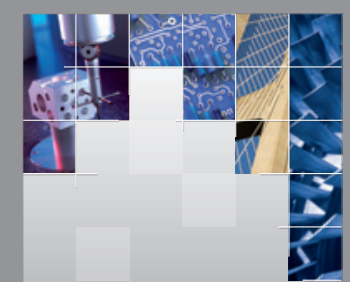

\section{Enfincering}
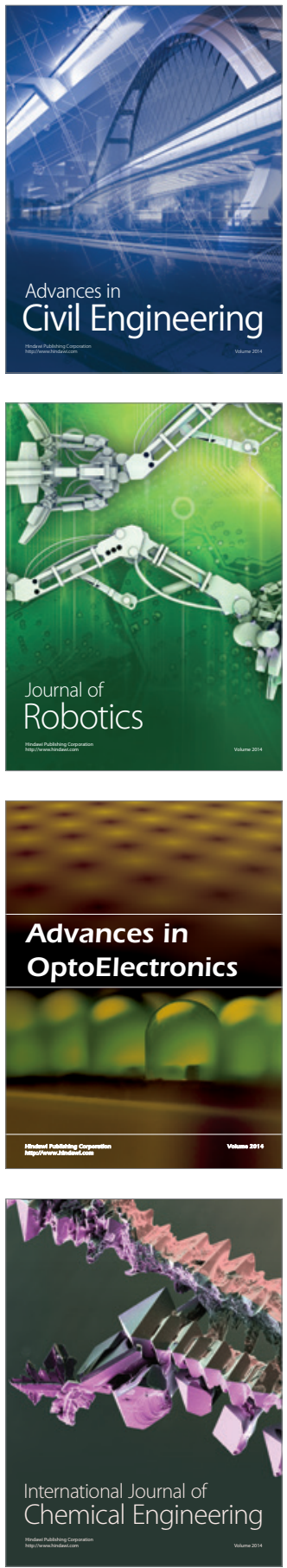

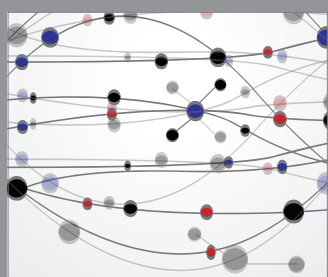

The Scientific World Journal

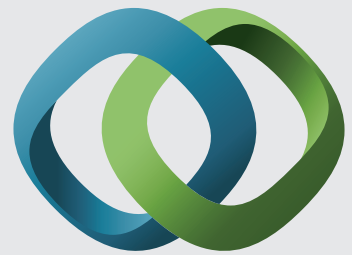

\section{Hindawi}

Submit your manuscripts at

http://www.hindawi.com
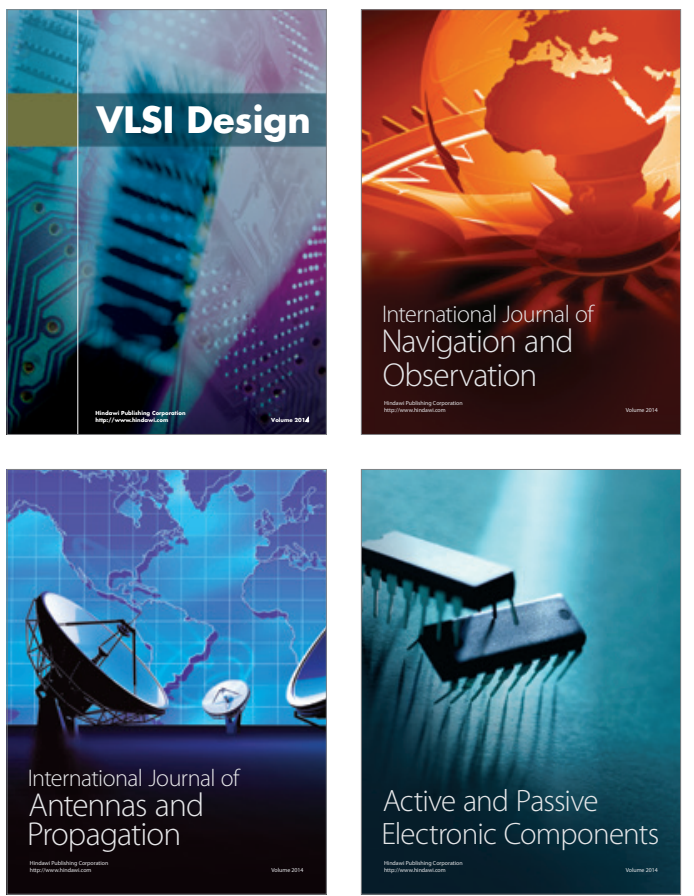
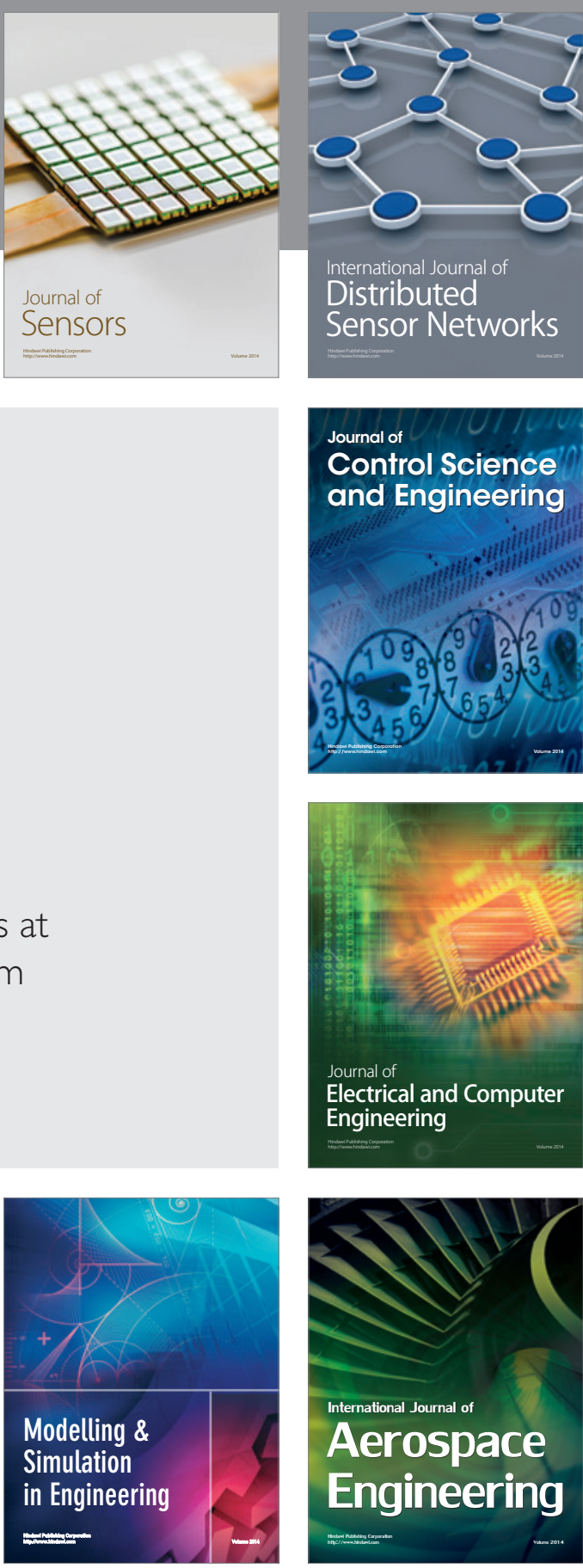

International Journal of

Distributed

Sensor Networks

Journal of

Control Science

and Engineering
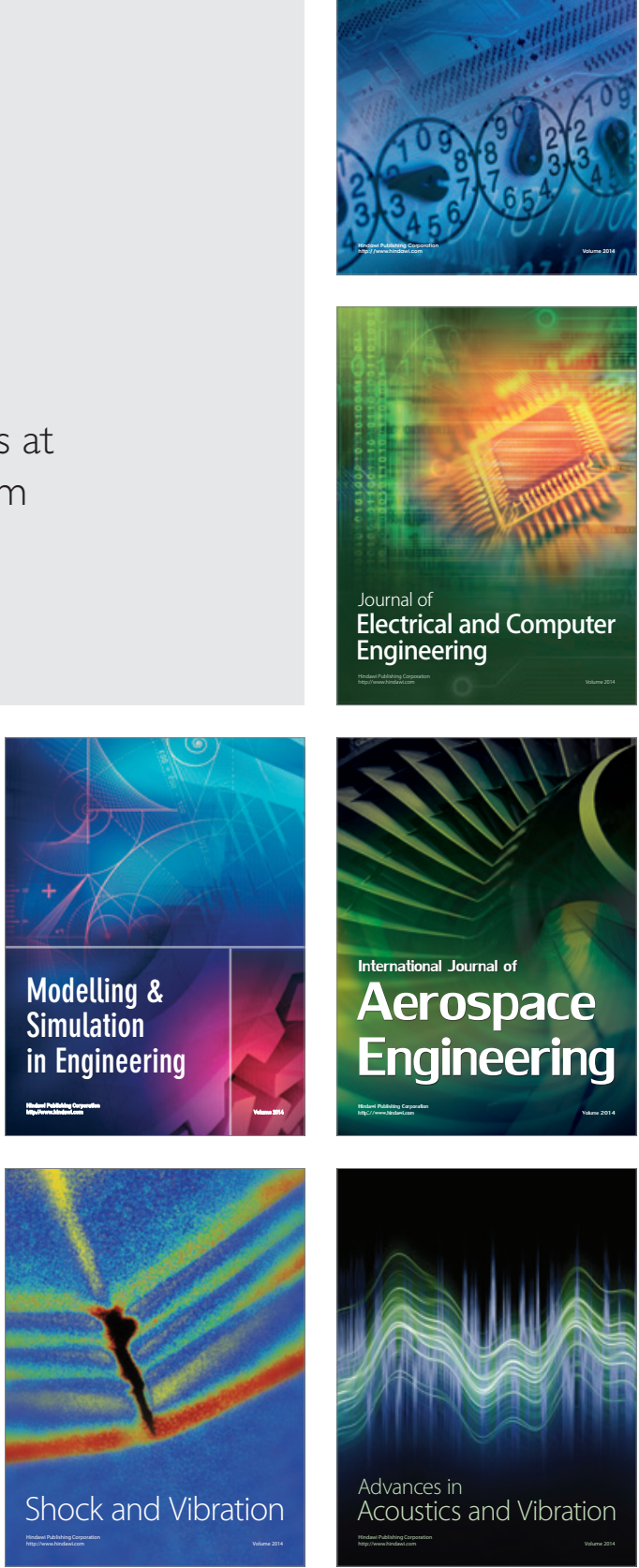